\title{
2016 von Neumann
}

Symposium

The Topological Recursion and its Influence

\section{in Analysis, Geometry, and Topology}

\author{
July 4-8, 2016, Charlotte, NC
}

The 2016 von Neumann Symposium on The Topological Recursion and its Influence in Analysis, Geometry, and Topology is organized by Motohico Mulase (Chair), University of California, Davis; Bertrand Eynard, Institut de Physique Théorique, CEA, and Chiu-Chu Melissa Liu, Columbia University, New York, and will take place at Hilton Charlotte University Place, Charlotte, North Carolina, July 4-8, 2016.

The topic was selected by the AMS von Neumann Symposium Committee, whose members at the time were J. B. Conrey, The American Institute of Mathematics; Michael J. Hopkins, Harvard University, and Terence Tao, University of California, Los Angeles.

Focus of Symposium. The proposed symposium is planned as a response to the recent extremely rapid and rich developments in the emerging research field generally known as the topological recursion. It has its origin in random matrix theory, and also in the work of Mirzakhani on the volume of the moduli space of hyperbolic surfaces. It has played a fundamental role in connecting seemingly unrelated areas of mathematics, such as matrix models, enumeration of Hurwitz numbers and Grothendieck's dessins d'enfants, Hitchin moduli spaces, the A-polynomials and colored polynomial invariants of knots, Gromov-Witten invariants, the WKB asymptotic analysis of 1-dimensional Schrödinger equations, and the non-Abelian Hodge correspondence. The symposium is planned at a time when many discoveries and crucial theorems have been established, yet numerous new mysteries are still arising.

The symposium will be organized around several plenary talks, four mini-courses, and fourteen to nineteen research talks. Details about

For permission to reprint this article, please contact: reprint-permission@ams .org.

DOI: http://dx.doi.org/10.1090/noti1299 the speakers and the program will be available on the website located at www.ams.org/meetings/ amsconf/symposia/symposia-2016. There will also be a series of shorter presentations.

The participation of qualified women, underrepresented minorities, junior scientists (advanced graduate students and recent $\mathrm{PhDs}$ ), as well as industry and national laboratory representatives is especially encouraged. To apply for an invitation to participate and to request limited support funds, please go to www.mathprograms.org and click on "as Program applicant." If you do not have a standing account, you must create one by filling in your email address, creating a password, clicking the button "this is my first login, please create an account for me," then clicking the "login" button. A login token will be sent almost immediately to the email address you provide. Return to www. mathprograms.org within twenty-four hours and click on the applicant login link to continue. After logging in, fill in the cover sheet and submit it; please ignore any instructions regarding reference letters. Click on "View Programs," then on "apply" for the von Neumann Symposium. Please follow the instructions listed on the site to complete your application. If you have questions while using this program, please contact the AMS at meet@ ams.org. The deadline for 2016 applications is March 1, 2016.

Participation in this program is limited. All requests will be reviewed and considered by the organizing committee. Letters of invitation with specific offers of support (if applicable) will be mailed in early April, along with a brochure of information, the program information known to-date, and information on travel and accommodation in Charlotte. All individuals who apply will be notified of the decision regarding acceptance or declination before May 1, 2016. 
Please note that those attending the Symposium should plan to arrive on Sunday, July 3, and depart on Saturday, July 9; lectures will be held Monday through Friday.

ADA Accessibility: It is the goal of the AMS to ensure that its conferences are accessible to all, regardless of disability. The AMS will strive, unless it is not practicable, to choose venues that are fully accessible to the physically handicapped. If special needs accommodations are necessary in order for you to participate in the von Neumann Symposium, please communicate your needs in advance to the AMS Meetings Department by:

- Registering early for the meeting

- Checking the appropriate box on the registration form, and

- Sending an email request to the AMS Meetings Department at mmsb@ams .org or meet@ams .org.

Welcoming Environment Policy: The AMS strives to ensure that participants in its activities enjoy a welcoming environment. In all its activities, the AMS aims to foster an atmosphere that encourages the free expression and exchange of ideas. The AMS supports equality of opportunity and treatment for all participants, regardless of gender, gender identity or expression, race, color, national or ethnic origin, religion or religious belief, age, marital status, sexual orientation, disability, or veteran status.

More details about this policy and how to address questions and/or make reports is posted at www.ams.org/about-us/governance/policystatements/we1coming-environment-policy.

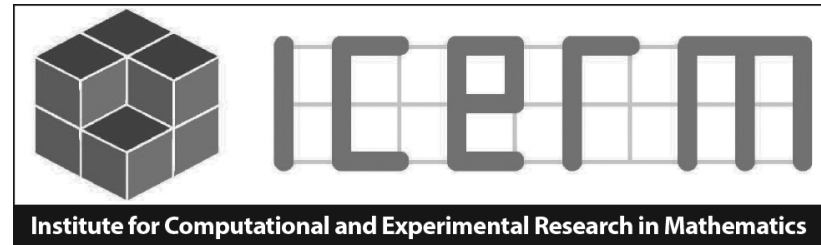

\section{SPRING SEMESTER 2017}

\section{Singularities and Waves in Incompressible Fluids}

January 30 - May 5, 2017

\section{Organizing Committee:}

Bernard Deconinck, University of Washington

Yan Guo, Brown University

Diane Henderson, Pennsylvania State University

Helena Nussenzveig Lopes, Federal University of

Rio de Janeiro

Govind Menon, Brown University

Paul Milewski, University of Bath

Walter Strauss, Brown University

Jon Wilkening, University of California - Berkeley

\section{Program Description:}

Incompressible fluids are an abundant source of mathematical and practical problems. The question of global-in-time regularity versus finite-time singularity formation for incompressible fluids, governed by the Navier-Stokes or Euler equations, has been one of the most challenging outstanding problems in applied PDE. There have also been new developments in the study of the onset of turbulence due to linear and nonlinear instabilities in incompressible fluids. Interfacial and surface water waves are physical phenomena that, in addition to the challenges outlined above, involve the evolution of free boundaries. These problems embody many of the mathematical challenges found in studies of nonlinear PDEs.

Topics of particular interest to be covered in three related workshops include: singularity formation, stability and bifurcation; the modeling and analysis of simplified phenomenological models for the description of coherent structures; and timedependent and steady free boundary problems including water waves, vortex sheets, capillary problems with contact lines and viscous waves with boundary layers. DETAILS AT:icerm.brown.edu.

Ways to participate:

Propose a:

- semester program

- topical workshop

- small group research program

- summer undergrad program

Apply for a:

- semester program or workshop

- postdoctoral fellowship

Become an:

- academic or corporate sponsor
About ICERM: The Institute for Computational and Experimental Research in Mathematics is a National Science Foundation Mathematics Institute at Brown University in Providence, RI. icerm.brown.edu
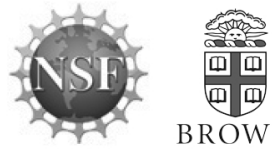